\title{
Experiencia Estética y Desarrollo Humano. Las Artes Temporales en la Génesis de Procesos Psicológicos Complejos
}

\section{Aesthetic Experience and Human Development. The Temporal Arts in the Genesis of Complex Psychological Process}

\author{
Silvia Español \\ Universidad de Buenos Aires \\ Consejo Nacional de Investigaciones Científicas y Técnicas
}

\begin{abstract}
En el área de la psicología del desarrollo se están generando nuevas hipótesis acerca de la génesis de la subjetividad e intersubjetividad infantil. Básicamente, se trata (a) de la detección de habilidades perceptuales infantiles muy tempranas ligadas al tiempo y (b) del reconocimiento de la presencia de antecedentes de las artes temporales (la música y la danza) en los primeros intercambios entre adulto y bebé. El propósito de este trabajo es presentar este modo naciente de acercamiento a la infancia y señalar su afinidad con lo que en filosofía de la mente se denomina "perspectiva de segunda persona".
\end{abstract}

Palabras Clave: intersubjetividad, teoría de la mente, artes temporales, segunda persona, desarrollo.

\begin{abstract}
In the field of developmental psychology new hypotheses are currently being brought forward about the infant's subjectivity and intersubjectivity. Namely, these are related to (a) the detection of very early perceptual abilities linked to time and (b) the recognition of the presence of antecedents of temporal arts (music and dance) in the mother-infant interactions. The purpose of this paper is to introduce this emerging approach to infancy and to outline its affinity with what is called "the second person perspective" in the field of philosophy of mind.
\end{abstract}

Keywords: intersubjectivity, theory of mind, temporal arts, second person, development.

La psicología vive en una permanente esquizofrenia epistemológica mirando con un ojo la cultura y con el otro la naturaleza, debatiéndose sin solución alguna entre la comprensión y la explicación, entre la interpretación y la experimentación. Sin embargo, está culturalmente viva. ¿Por qué? La respuesta que ensaya Blanco (2002) es que tal vitalidad depende de su capacidad para introducir criterios que permiten gestionar ideas de la subjetividad culturalmente viables. La psicología representa la idea de encontrar regularidades en el orden de la subjetividad y proporciona argumentos, a partir de cierta racionalidad, para legitimar, defender o desmontar las ideas o hipótesis sobre nuestra subjetividad.

La psicología del desarrollo, por su parte, apuntala desde una perspectiva evolutiva las diversas imágenes institucionalizadas del sujeto que las teorías psicológicas van brindando a la vez que, en oca-

Silvia Español, Facultad de Psicología, Universidad de Buenos Aires, Argentina. Consejo Nacional de Investigaciones Científicas y Técnicas (CONICET), Argentina. La correspondencia relativa a este artículo deberá ser dirigida a su autora. E-mail: silviaes@psi.uba.ar siones, analizando la génesis de los procesos psicológicos construye ella misma imágenes posibles. Hoy, la psicología cognitiva representa una de las máximas apuestas por establecer un conocimiento objetivo acerca de las raíces mismas de la subjetividad humana (Rivière, 1991). Y la psicología del desarrolo de corte cognitivista busca, y encuentra, mecanismos y representaciones innatos que sustentan y tornan posible una explicación mecanicista de la naturaleza humana. En la versión más fuerte de la psicología cognitiva se impone la idea de que aquello que podemos conocer, y a lo que debemos dedicar nuestra inteligencia, se restringe a capacidades concebibles como sistemas de cómputos, de naturaleza sub-personal e inaccesibles a la conciencia. Consecuentemente, la mente fenomenológica, aquella a la que tenemos un acceso directo, privilegiado, pero que es esencialmente inasible para el conocimiento científico, tiende a desfallecer por no cognoscible en el trastero de los misterios.

Pero nuestra disciplina está siempre en crisis, soportando antagonismos. En lo que respecta al problema de cómo los humanos llegamos a comprender que los otros son seres con mente o seres que po- 
seen estados mentales, la postura cognitivista tradicional considera que el conocimiento del mundo mental supone un proceso inferencialista-teórico basado en un modo particular de operar con representaciones. A ella se oponen abordajes que acentúan el valor de la experiencia subjetiva, interna e individual. Pero sobretodo, la faceta de la psicología que no renuncia al impulso a comprender e interpretar el fenómeno humano puede encontrarse en los trabajos que sugieren que la subjetividad, es decir, nuestro modo peculiar de concebir y orientarnos al mundo, a los otros y a nosotros mismos, emerge de las experiencias de intersubjetividad.

El propósito de este trabajo es presentar esta concepción actual de la intersubjetividad que se caracteriza por intentar desprenderse de la escisiones radicales entre mente y cuerpo y entre naturaleza y cultura impuestas por la concepción cognitivista, aunque prestando atención y haciendo uso de sus datos de laboratorio. Dos rasgos más la identifican: por un lado, la recuperación del enlace originario entre el ámbito psicológico y el estético, por otro, la confluencia de aproximaciones psicológicas y filosóficas.

A finales del siglo XIX, momento en que la psicología se reconoce como disciplina independiente, se inicia la transición teórica de la estética metafísica hacia la estética psicológica. Posiblemente, como sugieren Castro, Pizarroso y Morgade (2005), puede encontrarse allí el propio origen de la psicología ya que en cierto sentido el desplazamiento de la preocupación de lo estético al nivel del sujeto exigió que existiese la psicología. El proceso de psicologización del ámbito de lo estético conllevó la pregunta acerca del origen de la experiencia estética, y las artes temporales fueron concebidas como las artes primigenias (el término artes temporales abarca a aquellas artes en las que el tiempo es su factor constitutivo. La música y la danza son los casos paradigmáticos, ya que ellas son, respectivamente, las artes del sonido y del movimiento en el tiempo). Actualmente, se redefine el enlace entre el ámbito psicológico y estético de una manera interesante. Si el pensamiento decimonónico vinculó a las artes temporales con la experiencia emotiva de carácter empático, con la acción incontrolada e impulsiva y con los instintos más básicos y primitivos, fiel reflejo de la "animalidad" aún conservada en el sujeto humano -especialmente en los niños, en los pueblos primitivos y en algunas manifestaciones psicopatológicas-; en la actualidad, empieza a vislumbrarse la idea de que las artes temporales (la música y la danza) (a) participan en la génesis de funciones psicológicas complejas -como la adquisición del lenguaje, el reconocimiento de sí mismo y del otro y la capacidad de ficción-y (b) que conforman los primeros intercambios que se establecen entre el bebé y el adulto y se encuentran implicadas en las primeras experiencias de intersubjetividad. Se trata de un movimiento que sitúa a las artes temporales en la génesis de la subjetividad y que supone, además, que la gestación de la subjetividad se encuentra en experiencias de intersubjetividad primarias posibilitadas por el contacto corporal entre adulto y bebé y por la naturaleza musical de las interacciones tempranas. En este trabajo se expondrán las ideas principales concernientes al papel que las artes temporales cumplen en las experiencias de intersubjetividad y en la génesis del juego de ficción.

Como la propia psicología, esta propuesta cabalga entre la explicación y la comprensión, e incorpora aportes de la psicología cognitiva del desarrollo, de la estética naturalista y de la psicología de la música. El ámbito de la estética naturalista engloba aquellos trabajos que consideran que el dominio estético se encuentra ligado a los procesos de adaptación y evolución de la especie (Dissanayake, 2000a, 2000b, 2001; Tooby \& Cosmides, 2001). El área de la psicología de la música es extremadamente extensa y en ella se refleja la diversidad de problemas y posturas teóricas que caracterizan a la psicología en general y en particular a la psicología cognitiva (Algunos de las obras más representativas son: Deliege \& Sloboda, 1996; Imberty, 2002; Lerdahl \& Jackendoff, 1983; Wallin, Merker \& Brown, 2000).

Esta propuesta encuentra eco también en el ámbito filosófico. En realidad, el denominado problema de las otras mentes -la pregunta acerca de cómo es que podemos saber con certeza que los otros tienen sensaciones, sentimientos, ideas si tan sólo tenemos acceso a nuestros propios estados mentales y no podemos observarlos en los otros- es ciertamente una cuestión filosófica de larga data. Desde hace unas décadas, en el área de la filosofía de la mente, la cuestión se reedita bajo el término Folk Psychology, indisolublemente entramado con las investigaciones psicológicas en el área del Sistema de Teoría de la Mente. En estos ámbitos se desarrolla el enfrentamiento entre dos posturas: la Teoría de la Teoría y la Teoría de la Simulación, las cuales, como se verá más adelante, pueden caracterizarse como perspectivas de tercera y de primera persona, respectivamente. La naciente concepción de 
intersubjetividad también emerge en el pensamiento filosófico y va cobrando cuerpo en lo que empieza a denominarse Perspectiva de Segunda Persona (en clara alusión a su diferencia con las otras dos). También aquí puede observarse el entramado con las discusiones psicológicas aunque, en este caso, el diálogo no es completo. Probablemente ello se deba a que los aportes provenientes de la psicología de la música y de la estética naturalista son poco conocidos incluso en la propia psicología.

A continuación, comentaré brevemente las dos aproximaciones clásicas al problema de la atribución mental -la Teoría de la Teoría y la Teoría de la Simulación- para contraponer a ellas la naciente Perspectiva de Segunda Persona. Señalaré, luego, los aportes clásicos de la psicología del desarrollo que pueden considerarse acordes a la Perspectiva de Segunda Persona. Una vez hecho esto, me adentraré en la descripción de las nuevas hipótesis y observaciones acerca de las experiencias infantiles provenientes del diálogo establecido entre la psicología del desarrollo, la psicología de la música y la estética naturalista. Finalmente, me centraré en mostrar que dichas hipótesis y observaciones, además de permitir un abordaje más cabal de las experiencias de intersubjetividad acorde a la Perspectiva de Segunda Persona, tornan posible también reinterpretar el desarrollo ontogenético de algunas capacidades cruciales infantiles, como la capacidad de ficción.

\section{La Perspectiva de Segunda Persona y las Experiencias de Intersubjetividad}

La discusión acerca de cómo comprendemos que los otros son seres con mente, cómo somos capaces de atribuir estados mentales a nuestros congéneres y a nosotros mismos, clásicamente se dirime entre dos aproximaciones: la Teoría de la Teoría y la Teoría de la Simulación. En la primera, se sostiene la idea de un acceso inferencial-teórico al mundo mental, propio y ajeno, y se postula que nuestro conocimiento de otras mentes se debe a operaciones de mecanismos que concentran el conocimiento acerca de la subjetividad de manera similar a las teorías científicas. Se supone la existencia de representaciones de una representación atribuida a otro sujeto es decir, de metarrepresentaciones (Gopnik \& Meltzoff, 1997/ 1999). La otra postura, en cambio, cuestiona que sean habilidades de naturaleza teórica las que subyacen a las capacidades mentalistas y supone que lo que está en juego son procesos de acceso interno a la propia mente y proyección simulada en la de los demás. Desde este enfoque (bien representado por Goldman, 1993, 2000 y Harris, 1993, 2000/2005), se considera que es el acceso privilegiado a la primera persona del singular, primariamente experiencial y no teórico, lo que permite mediante su proyección simulada en los demás el acceso al mundo mental del otro. Es la experiencia inmediata de lo mental que el sujeto realiza en el recinto irremediablemente privado de la primera persona del singular la que permite dar el salto hacia las otras mentes vía la simulación (para una caracterización de ambas posturas puede verse Rivière, 1997/2003 y Sperber, 2000).

En otras palabras, la cuestión se dirime entre una perspectiva de primera persona -en la que el enclaustrado mundo de la subjetividad es la condición necesaria para poder acceder la subjetividad de los otros- y una perspectiva de tercera persona en la que la atribución de mente a otros se restringe a inferencias acerca de un otro representado con el cual no es requisito establecer ningún tipo de contacto interpersonal. Ambas coinciden en considerar que el proceso de adquisición de las habilidades mentalistas maduras consiste en una secuencia que va desde la comunicación intencionada preverbal, a partir del último trimestre del primer año de vida, al juego de ficción en el segundo año; y desde la comprensión de deseos en el tercer año de vida a la comprensión de creencias falsas en el cuarto. Ambas también suelen reconocer la importancia que tienen las experiencias de intersubjetividad que ocurren durante los primeros meses de vida.

En el ámbito filosófico, desde lo que empieza denominarse Perspectiva de Segunda Persona (Gómez, 1996; Gomila, 2003; Scotto, 2002; Thompson, 2001), se esgrimen algunas críticas que engloban a las dos aproximaciones reseñadas. Se afirma que ambas se han restringido a los rasgos epistémicos y a una concepción reflexiva de la atribución mental. En la versión restrictiva de la teoría de la mente como atribución de actitudes proposicionales, y en su formulación metarrepresentacional, es evidente la apuesta por una comprensión reflexiva de los conceptos mentales involucrados, pero también el proceso de simulación parece concebirse como un proceso consciente, reflexivo y propositivo. Es decir, en ambos casos la atribución mental parece implicar una comprensión reflexiva de los conceptos mentales involucrados. Por otro lado, se señala que cuando se ocupan de las emociones las conciben como estados mentales internos y las tratan siempre de una manera unilateral. La Perspectiva de Segunda Persona, en cambio, no considera que los contactos 
interpersonales supongan necesariamente una comprensión teórica de que los otros son seres con estados mentales internos e inobservables ni que requieran tampoco un acceso directo e intuitivo al mundo mental propio y privado y una posterior simulación proyectada en los demás; al contrario, supone que los modos básicos de estar y comprender a los otros, y a uno mismo, se basan en un conjunto de habilidades para la comprensión recíproca que se desarrolla y expresa en contextos interactivos, por ende, públicos y sociales. Se asume que en la vinculación con el otro intervienen componentes que tienen una directa expresión pública, como expresiones faciales o posturales y diversos patrones conductuales. Y se considera que los aspectos expresivos -como el tono de voz o la configuración facial-son percibidos como directamente significativos y constituyen la base de una reacción correspondiente que tiene pleno sentido dentro de la situación de una interacción que se despliega en el tiempo. Lo que un sujeto hace tiene una respuesta sensible en el otro, de tal suerte que acciones y reacciones tienen los rasgos de la reciprocidad. Se destaca también que la atribución mental en contextos interactivos no sólo es recíproca sino que además los participantes se percatan de su mutua atribución, lo cual condiciona el contenido de la atribución. Lo mental no es por tanto considerado algo privado sino público, expresivo y dinámico (Gomila, 2003; Scotto, 2002).

La Perspectiva de Segunda Persona parece novedosa en filosofía. Scotto (2002) señala los pocos casos en los que puede reconocérsela -el trabajo de Gómez (1996) el de Gomila (2003) y el libro editado por Thompson (2001) - y ubica sus antecedentes en el pensamiento de Wittgenstein y en la tradición fenomenológica. Pero en psicología la situación no es la misma: la mayoría de los trabajos que desde la década del 70 se realizan sobre las interacciones tempranas adulto-bebé y la comunicación preverbal son acordes a la perspectiva de segunda persona. Estos estudios han arrojado luz sobre los primeros modos de "estar y hacer con el otro". Es en este ámbito en el que se asienta el término intersubjetividad en psicología (Trevarthen, 1982), afirmándose que a través de las interacciones atencionales y emocionales con los otros, el bebé, aproximadamente desde los dos meses de vida, participa en experiencias de intersubjetividad primaria en las que comparte estados emocionales con los otros. Las protoconversaciones (Bateson, 1979), las reacciones circulares sociales (Rivière, 1987, 1999/
2003) y la imitación neonatal que torna posibles los tempranos juegos de imitaciones mutuas (Kugiumutzakis, 1998; Trevarthen, 1998) son algunos de los hallazgos más importantes que muestran que, desde el inicio, el bebé es un ser social que interactúa organizadamente con los otros. Sin embargo, es preciso destacar que el ámbito de análisis de estas investigaciones se ha restringido, casi siempre, al primer año de vida.

Actualmente, la mayoría de los investigadores que trabajan en la ontogenésis del Sistema de Teoría de la Mente han hecho un voto de aceptación del papel genético de los estados primarios de intersubjetividad. En general, suele ponerse el acento en dos capacidades tempranas infantiles: (a) la capacidad de expresar emociones (las expresiones emocionales darwinianas clásicas: miedo, ira, alegría, tristeza, interés, disgusto, etc.) y (b) la capacidad de imitación neonatal. Algunos lo hacen respetando los rasgos de la Perspectiva de Segunda Persona (Gómez, 1998; Hobson, 1993/1995; Rivière, 1999/ 2003), otros, transforman la explicación de las experiencias de intersubjetividad dándoles el aroma mentalista típico del enfoque cognitivista (Gopnik \& Melzoff, 1997/1999) [en Beebe et al. (2003) puede encontrarse un análisis de las diferentes acepciones de intersubjetividad].

A continuación, se presentarán las aportaciones provenientes del diálogo establecido entre la psicología del desarrollo, la psicología de la música y la estética naturalista. Como podrá observarse, ellas muestran otra manera de comprender el mundo emocional $\mathrm{y}$, aunque reconocen el papel esencial que cumple la imitación en el establecimiento de las experiencias de intersubjetividad, acuñan un concepto muy cercano al de imitación -el entonamientoesencial para el despliegue de las mismas. Ellas modifican también nuestro modo de entender las interacciones tempranas que pasan de ser concebidas como protoconversaciones a ser vistas como performances. Cada una de estas aportaciones denota una concepción no escindida entre lo corporal y lo mental: los elementos con los que se describen los intercambios entre adulto y bebé son componentes mentales que tiene una expresión pública directa o, lo que es lo mismo, son movimientos corporales que se perciben como directamente significativos. A su vez, los actos de cada uno de los miembros de la díada son concebidos como una ocasión para una reacción correspondiente que se despliega en el tiempo y que tiene los rasgos de la reciprocidad. Es decir, a cada una de las aportaciones que se 
presentan a continuación subyace la asunción de una Perspectiva de Segunda Persona.

\section{La Perspectiva de Segunda Persona y las Artes Temporales}

En los estudios de interacción temprana, la temporalidad de la interacción fue siempre un concepto central. Hace varias décadas, se destacó, por ejemplo, que la interacción de la díada está organizada temporalmente desde el inicio, adoptando la forma de alternancia o sincronía interactiva y se mostró cómo la manipulación experimental del tiempo de la interacción produce inmediatas respuestas de malestar en el bebé (Murray \& Trevarthen, 1984). Pero en la actualidad, se acentúa el papel del tiempo en el desarrollo ontogenético. La detección de habilidades perceptuales infantiles muy tempranas ligadas al tiempo así como la observación de rasgos prototípicos de las artes temporales presentes en los primeros intercambios entre adulto y bebé permiten expandir la comprensión de las experiencias de intersubjetividad. De tal suerte, el mundo emocional que adscribimos al infante se extiende hacia los denominados sentimientos temporales y se concibe la idea de que lo primero que los adultos ofrecen a los bebés es una especie de espectáculo o performance multimedia a la cual los bebés responden con sus capacidades precoces de interacción interpersonal.

\section{Los Sentimientos Temporales}

Tendemos a pensar que la experiencia estética es algo del orden de lo inefable que requiere de cierta sofistificación de la sensibilidad sólo posible en la adultez. Lo cual es razonable. Sin embargo, al menos en lo que respecta a las artes temporales, los cimientos de la experiencia estética parecen encontrarse en los momentos más tempranos del desarrollo humano.

La psicología del desarrollo de corte cognitivista ha brindado algunos datos cruciales al respecto. Por ejemplo, ha detectado la capacidad inicial de los bebés, desde los primeros meses de vida, de establecer equivalencias transmodales de rasgos muy globales de las estimulaciones que reciben provenientes de diversas modalidades sensoriales (Stern, 2000). Transmodalidad significa tránsito de información de una modalidad sensorial a otra. Algo a lo que -de acuerdo con Stern (1985) - las artes nos invitan frecuentemente: en los espectáculos de luz sinfónica, en la integración de sonido-visión en el cine y fundamentalmente en la danza en la que el sonido y el movimiento se funden en el tiempo. Nuestra capacidad de percepción transmodal nos permite aunar diversidad de sensaciones provenientes de diferentes modalidades y establecer equivalencias entre ellas. La percepción transmodal, que se vincula con formas de arte sofisticadas (como la poesía de los simbolistas franceses del XIX, "olores frescos como la piel de un niño" escribe Baudelaire), está en la base de la experiencia inicial infantil.

Los bebés pueden establecer equivalencias transmodales extremadamente globales vinculadas a rasgos de la estimulación que reciben a los que son especialmente sensibles, como a los rasgos temporales: en especial la duración, los ritmos simples y los contornos temporales, es decir, los cambios en el tiempo de la intensidad de la sensación (Stern, 2000). La capacidad de percepción transmodal de rasgos temporales subyace a una de las preferencias estimulares más importantes detectadas en el laboratorio experimental del psicólogo: a los bebés los atrae especialmente la estimulación concordante. Cuando, por ejemplo, se les presenta estímulos muy simples como (a) una esfera que se mueve y un sonido sincrónico de igual duración y (b) una esfera que se mueve y un sonido desincronizado y con una duración diferente; los bebés miran más tiempo, prefieren, la estimulación concordante, es decir, la estimulación apareada temporalmente (Lewkowicz, 1992, 2000, 2002)

En situaciones artificiales, los bebés detectan y prefieren estímulos concordantes, equivalentes tansmodalmente en algún rasgo temporal. Este es un dato preciso, seco y aislado. Pero cuando esta capacidad se pone en juego en intercambios humanos el panorama cambia. La capacidad de percepción transmodal de rasgos temporales permite una primera organización del mundo interpersonal del infante. El bebé puede aunar, por ejemplo, las sensaciones táctiles y auditivas que recibe cuando su madre emite sonidos, palabras u oraciones de una intensidad, una duración y un ritmo semejantes a las palmadas suaves que le da en los brazos y a los movimientos oscilantes de su cabeza hacia atrás y adelante. La misma información temporal de duración y ritmo, el mismo perfil de intensidad se le muestra una y otra vez a través de diferentes modalidades sensoriales. Y esto ocurre no solo de un modo benevolente: de la misma manera que si los sonidos del habla materna son regulares, pianos y cadenciosos probablemente también sea así el modo en que lo 
toca, si el sonido de su voz es abrupto, forte y disruptivo posiblemente así sea también el modo que lo toca. Surgen así las experiencias más básicas de "estar con el otro", aquellas en la que se comparte la experiencia del tiempo.

Los sentimientos temporales que acontecen en la díada adulto-bebé no se entienden en términos de las emociones darwinianas clásicas como la alegría, la tristeza, el miedo, el disgusto, la ira, etc. El fluir de intercambios de expresiones, movimientos y sonidos pautados temporalmente permite compartir patrones temporales e implantar una sintonía mutua de sentimientos dinámicos (Trevarthen, 1998, 2000) que sólo pueden describirse mediante términos dinámicos como agitación, desvanecimiento progresivo, fugaz, explosivo, crescendo, decrescendo, estallido, dilatado. Stern $(1985,2000)$, quien reconoce basarse en las ideas de Langer (1967), define los sentimientos temporales como perfiles de activación en el tiempo, como cambios pautados de la intensidad de la sensación y del tono hedónico en el tiempo. Y distingue entre los sentimientos temporales y las emociones clásicas: los sentimientos temporales pueden acompañar a las emociones darwinianas clásicas (la tristeza puede ser fugaz o dilatada, acelerarse o desvanecerse al igual que la alegría) pero también presentarse sin ellas en cualquier tipo de acto humano. Así, el levantarse de una silla puede ser explosivo o dilatado, y fugaz el gesto y evanescente la caída del recuerdo.

Los sentimientos temporales son esencialmente una experiencia transmodal, una experiencia en la que se aúnan la diversidad de sensaciones provenientes de diferentes modalidades en función de su perfil de activación, es decir, de las variaciones de la intensidad de las sensaciones. En la adultez, de la diversidad de sensaciones provenientes del extensísimo espectro de toda nuestra experiencia. "Un ataque de cólera o alegría, un torrente percibido de luz, una secuencia acelerada de pensamientos, una ola de sentimientos evocados por la música y una toma de narcóticos, pueden sentirse por igual como irrupciones (...)"'(Stern 1985/1991, p. 77). En la infancia, de la diversidad de perfiles de activación provenientes de todas las modalidades -táctil, visual, auditiva, kinestésica- que el bebé experimenta en sí mismo y en los otros, en actos cotidianos y simples. De acuerdo con Stern (1985), lo que experimenta el infante en su mundo interpersonal, especialmente desde el nacimiento hasta los seis meses, se asemeja a lo experimentamos al escuchar música o al ver un espectáculo de danza abstracta.
Stern (1985) considera que los sentimientos temporales son difícilmente expresables a través del lenguaje; aunque el lenguaje poético puede evocarlos, son las artes temporales quienes los expresan con maestría. Pero también señala que existe un modo básico o primitivo de referirlos: el entonamiento. El entonamiento específicamente se refiere a algo que hacen las madres en relación con las conductas de sus bebés. Se trata de alguna forma de apareamiento, frecuentemente transmodal, de la intensidad, la pauta temporal o la pauta espacial de alguna conducta del bebé. No se trata de una imitación, de la traducción fiel de la conducta abierta, sino que la madre toma algo de una expresión del bebé y lo transforma en otra cosa, cambiándole la modalidad. Aparea, por ejemplo, sus vocalizaciones con los cambios de intensidad en el tiempo (por ejemplo, aceleración- desaceleración) de los movimientos del bebé; o si el niño golpea un muñeco estableciendo un ritmo constante, cae en ese ritmo pero en otra modalidad, por ejemplo la vocal, o aparea la duración de la vocalización del bebé con la duración de la caricia de sus manos. Se crean así pequeñas "analogías" entre gestos, sonidos y movimientos corporales. En palabras de Imberty (2002), la madre intenta encontrar aquel "color" o "tonalidad", percibidos y compartidos, utilizando toda la capacidad de transposición transmodal que el bebé posee. Y al hacerlo lleva el foco de atención a lo que está detrás de la conducta, al carácter del sentimiento que se está compartiendo, refundiendo la experiencia emocional en otra forma de expresión.

\section{La Elaboración Temporal, Dinámica y Transmodal de la Estimulación Materna}

Las personas, al menos en amplios sectores de la cultura occidental, no nos portamos de la misma manera cuando estamos con bebés que cuando estamos entre adultos. El modo en que hablamos y las cosas que hacemos son, si las miramos distanciadamente, casi extravagantes. Cuando nos dirigimos a los bebés, los adultos, entre otras cosas, remarcamos el pulso, retardamos el tiempo del habla, hacemos pausas más largas y hablamos más rítmicamente, con frases bien segmentadas, con una métrica repetitiva regular en la que predominan la repetición de estrofas y las aliteraciones (Dissanayake, 2000a, 2000b; Dissanayake \& Miall, 2003). También la melodía de nuestra habla se transforma: tendemos a usar un conjunto de cinco o seis prototipos melódicos. Usamos melodías ascendentes para llamar la atención 
del bebé o para darle turno en un diálogo y contornos descendentes ante la sobrexcitación del niño o para finalizar el diálogo (Papôusek, 1996). Las transformaciones del habla encuentran eco en los bebés que parecen estar biológicamente presintonizados y dispuestos para procesar los sonidos del habla (prefieren estímulos de la longitud y frecuencia de onda que caracterizan la voz humana y específicamente prefieren la voz materna a otras (Rivière \& Sotillo, 1999/2003). La sensibilidad y la preferencia infantil hacia los sonidos del lenguaje está vinculada con la sensibilidad a los rasgos musicales presentes en el habla como los contornos melódicos y tímbricos de la vocalización parental y sus atributos rítmicos (Treuhb, 2000).

Pero los sonidos del lenguaje son sólo una parte de la rica estimulación que los adultos ofrecen al bebé. De acuerdo con Dissanayake (2000a, 2000b), el extenso período de neonatenia de la cría humana creó una presión selectiva para la proximidad psicológica y para los mecanismos cognitivos que aseguraran un extenso y mejor cuidado maternal. Fue el motivo de una específica adaptación humana -la elaboración- de las conductas filiativas presentes en otros primates: expresiones faciales, gestos y sonidos. La elaboración no es más que el moldeado dinámico, rítmico y transmodal de dichas conductas que tiene la virtud de conducir directamente a un estado de mutualidad inherentemente placentero. La estimulación materna tal como la cualifica Dissanayake (2001), semeja una performance multimedia, ya que las emisiones peculiarmente estereotipadas o ritualizadas se acompañan de exageradas expresiones faciales y de movimientos del cuerpo. El bebé recibe una estimulación que está modelada dinámica, rítmica y transmodalmente a través de todas las modalidades disponibles -visual, auditiva, táctil, kinestésica-. Ciertamente no se trata de una performance en el sentido de un espectáculo a ser contemplado ya que, como se ha destacado hace tiempo (Nadel, 1996; Rivière, 1987/2003), la conducta materna es contingente a las respuestas del bebé. Se trata de una contingencia no rígida sino imperfecta que atrae poderosamente la atención del bebé que responde con vocalizaciones, movimientos sonrisas y expresiones faciales y que incitan a los padres a continuar la interacción entrando en lo que se denomina "reacciones circulares sociales". En ellas no hay un plan predeterminado, como en las performances, sino que se trata de un hacer conjunto en el que intervienen los componentes de las artes temporales (Español, 2006, en prensa), como los entonamientos, las modificaciones en la dinámica del sonido y del movimiento y la repetición de pautas de comportamiento con sutiles variaciones.

Hace tiempo se señaló que la forma repeticiónvariación es un rasgo esencial de la estimulación materna. La madre despierta la expectativa del bebé a través de la elaboración de estímulos que cambian de una forma interesante y no totalmente predecible. Repite frases, movimientos, sonidos, expresiones faciales exagerando sus contornos melódicos, su amplitud, duración y las pausas entre ellos; pero no siempre de manera idéntica sino realizando variaciones en la intensidad, el ritmo, el tono de forma tal que la estimulación es, al mismo tiempo, conocida pero nueva. Un mismo juego, como ascender con los dedos por la panza del bebé hasta terminar haciéndole cosquillas en el cuello, se realiza repetidamente variando alguno de sus elementos, la velocidad de los dedos o la demora antes de la excitación final, o acompañándolo de vocalizaciones o exagerando la expresión facial. La forma "variaciones sobre un tema" es un tipo de estimulación ideal para el infante: (a) porque si fuese siempre igual el infante se habituaría y perdería interés; y (b) porque la repetición genera una regularidad que permite anticipar el curso del tiempo, es decir le permite predecir lo que vendrá (Rivière, 1987/2003). Como señala Dissanayake (2001), la manipulación lúdica de la forma repetición-variación crea una anticipación particular, basada en la incertidumbre, la sorpresa y en la gratificación demorada de la expectativa. Imberty (2002) vincula la forma repetición-variación de las interacciones tempranas con la forma musical y sugiere que la repetición musical, al igual que la repetición de las secuencias comportamentales, genera el tiempo, un presente que va hacia algo, un antes y un después, con los cuales el compositor invita al auditorio (y el adulto invita al infante) a recordar y anticipar, con un margen suficiente de incertidumbre a fin de que cada vez se insinúe la sensación de que la repetición podría haberse no realizado o que la misma expectativa puede fundirse en otra, la cual a su vez puede también no ser completamente diferente.

\section{Las Artes Temporales en la Génesis del Juego de Ficción}

Aunque en los trabajos reseñados suele afirmarse que las experiencias de intersubjetividad temprana son el cimiento del desarrollo posterior de diversas habilidades, lo cierto es que las investigaciones sobre las experiencias de intersubjetividad se han 
abocado fundamentalmente al estudio del primer año de vida del niño. Asimismo, si bien los trabajos clásicos sobre las experiencias de intersubjetividad están en contacto con los estudios en el Sistema de Teoría de la Mente, hasta donde tengo conocimiento, a excepción del trabajo de Dissanayake (2001), no existe intercambio con las investigaciones que incorporan el área de la psicología de la música y el de la estética naturalista. En lo que sigue procuraré aportar algunos datos observacionales que indican que los antecedentes de las artes temporales que cualifican las tempranas interacciones entre el bebé y el adulto entraman (durante el segundo y tercer año de vida) la génesis del juego de ficción, uno de los precursores del Sistema de Teoría de la Mente.

Para que podamos actuar, pensar y comunicarnos sobre acontecimientos que no están atados a la realidad inmediata, para que, en última instancia, pueda emerger la imaginación o la ficción, es preciso poder saltar la realidad, es necesario poder distanciarse tanto de lo que los sentidos nos muestran aquí y ahora como de la realidad convencionalmente construida. Es posible que el desprendimiento de lo que los sentidos nos muestran aquí y ahora se inicie, tal como considera Dissanayake (2001), en el período eminentemente diádico de intersubjetividad primaria gracias a la forma repetición-variación. $\mathrm{Su}$ hipótesis es que la variación dentro de la repetición lleva a que el bebé se ejercite en la comparación y en la evaluación de las diferencias y discrepancias con lo esperado. Actualmente, todo lo que está vinculado con procesos psicológicos desprendidos de la realidad inmediata, relacionados con la imaginación, cae bajo el rótulo de cognición desacoplada. Dissanayake (2001) sugiere que quizá el ejemplo más temprano de cognición desacoplada, es decir, la primera instancia de la imaginación, surja cuando el bebé compara una variación de una conducta con lo que esperaba y reconoce y aprecia simultáneamente su similitud y diferencia.

Pero el representante prototípico de la cognición desacoplada es el juego de ficción infantil; una actividad que denota la posibilidad de desprenderse de lo que los sentidos nos muestran pero también de la realidad convencionalmente construida.

El desarrollo del juego de ficción implica fundamentalmente trasformación de la acción (Español, 2004). Alrededor de los doce meses los niños aprenden en colaboración con los adultos a utilizar instrumentos relacionados con sus actividades básicas (comer con cuchara, beber de un vaso) y, casi al mismo tiempo, comienzan a realizar con ellos juegos funcionales: los usan de modo descontextualizado, sin que su acción tenga los efectos que tendría de realizarse efectivamente. En primates no humanos también pueden observarse conductas de engaño y simulaciones de acciones -como comer-en ausencia de los objetos que las soportan así como usos descontextualizados de objetos -como hacer que se bebe de una taza vacía- (Gómez \& Martín-Andrade, 2002). Pero la ficción supone no sólo "simular" algo mediante un uso descontextualizado de objetos, o realizar la acción en vacío, sino que implica quebrar lo aprendido, transformar radicalmente los significados convencionales de las acciones y los modos de usos de los objetos, haciendo que algo sea otra cosa (como cuando un palo se transforma en un peine, en una muñeca o en un avión, o cuando una pinza es alimentada con fichas de plástico). El tránsito desde la descontextualización hacia la operación con representaciones desacopladas en el juego de ficción supone lo que frecuentemente se denomina "proceso de ritualización”. Éste es, por tanto, el germen de la capacidad ficcional específicamente humana. $\mathrm{Si}$ se analiza el proceso de ritualización a la luz de la incidencia de las artes temporales puede observarse un paulatino proceso de extrapolación o exteriorización de los antecedentes de las artes temporales que ocurrían en las relaciones diádicas tempranas.

En diferentes estudios observacionales (Español, 2005, 2006, en prensa; Shifres \& Español, 2004), hemos detectado que durante el segundo año de vida se inicia una extrapolación de la elaboración de la estimulación materna a la propia acción infantil. Como en tantos otros aspectos del desarrollo, aquello que en principio el niño percibe un tanto pasivamente, pasa a formar parte de su propia actividad. Así, en el proceso de ritualización puede observarse la "extrapolación" o apropiación de la forma repetición-variación, del entonamiento y del moldeado dinámico-temporal de las acciones. Por ejemplo, en las pequeñas "narraciones en acción" que los niños construyen combinando usos descontextualizados de objetos (como cucharas, platos vasos), que contienen varios actores (el niño, el adulto, los muñe$\cos )$ que pueden ser posibles receptores o agentes de la acción, empiezan a incluirse sonidos (como "shhhh" al servir de una tetera vacía, o el "aaamm" al llevarse una cuchara vacía a la boca). Estos sonidos son apareamientos de la pauta temporal del movimiento realizado, es decir, son entonamientos activamente realizados por el niño. También los niños acentúan los cambios en la dinámica del movimiento implicada en la acción que se acelera o retar- 
da, se abrevia o exagera. Asimismo, la forma repetición-variación parece sostener las combinaciones de esquemas de acción que empiezan a realizarse de una manera fija y repetida pero que, sin embargo, soportan la inclusión de pequeñas variaciones (peinar y poner perfume a la muñeca siempre del mismo modo hasta que (imitando el comportamiento de un adulto) se incorpora una inspiración exagerada. Estos mismos elementos participan también en la creación de los personajes ficcionales. Por ejemplo, cuando una niña a los dos años y medio "hace de monstruo" adopta un gruñido peculiar que repite junto con un movimiento amplio y exagerado de sus manos puestas en forma de garra, que entona con la duración del sonido, y lo hace en el marco de una estructura que se repite rítmicamente asociada a acercarse y alejarse de la persona a la que asusta. Es posible, por tanto, que la elaboración apoye el proceso de transformación de la acción o, en otras palabras, que la ficción subsuma componentes de las artes temporales.

Por otro lado, el juego de ficción se vincula con lo que se denomina Juego Musical. De acuerdo con Merker (2002), la regulación temporal inicial de la díada no es musical sino que se restringe a la regulación temporal basada en el tiempo de reacción y en la familiaridad. La regulación del tiempo musical se basa en la subdivisión igual del tiempo a través del pulso musical. Este tipo de timing basado en una pulsación subyacente es el mecanismo fundamental de los desempeños musicales. Sin embargo, durante el segundo año de vida, los niños desarrollan un mecanismo de timing conductual basado en el pulso subyacente que se ejercita en la infancia a través del Juego Musical en el que, a través de las acciones de la madre, el niño ajusta su timing a un tercer miembro: la estructura métrica de la canción o el juego. El juego musical se presenta a veces de forma aislada pero también en contextos de juego de ficción. Cuando esto ocurre, suele seguir la secuencia "juego de ficción-juego musical-juego de ficción". En la escena de ficción aparece algún elemento disparador por el cual la atención de la díada pasa de la temática de ficción al componente musical (por ejemplo, la repetición de algún patrón rítmico utilizando un objeto para percutir). En estos casos, el objeto que suscita el juego musical pierde la función que venía cumpliendo durante el juego de ficción y pasa a ser simplemente un agente del juego musical. Al agotarse el juego musical frecuentemente se retorna a la temática del juego de ficción y éste incorpora algunos atributos del juego musical previo. Por ejem- plo, el pattern rítmico del juego musical -temáticamente abstracto- se adhiere a la acción ficcional de marcar en una caja de madera como si fuese un teléfono. Cuando el juego musical irrumpe en el juego de ficción, desplaza su contenido temático y lo reemplaza por acciones que parecen dejar en la mente del infante una suerte de "significado flotante" (Cross, 2003), el cual apoyaría luego la organización temporal y el despliegue temático de la escena ficcional.

\section{Conclusiones}

En este trabajo se ha pretendido mostrar la conexión que existe entre propuestas que provienen de disciplinas o ámbitos diferentes y que incluso en algunos casos se desconocen entre ellas. La idea que las aúna es que la subjetividad humana se constituye a partir de experiencias de intersubjetividad, y que estas últimas emergen en situaciones de interacción social que se despliegan en el tiempo. Se ha indicado también la emergencia de un movimiento que sitúa a las artes temporales en la génesis de las más tempranas experiencias de intersubjetividad. $\mathrm{Y}$, en sintonía con este movimiento, se ha extendido el análisis de la incidencia de las artes temporales hacia a la ontogénesis del juego de ficción.

Como habrá podido observarse, estas propuestas se caracterizan por hacer uso de los datos experimentales provenientes de la psicología cognitiva pero situándolos en el horizonte de la reflexión comprensiva y de la interpretación. En conjunto, muestran que mirar la experiencia humana desde las artes puede ayudar a reinterpretar el proceso ontogenético y ensayar nuevas hipótesis acerca de temas centrales de la psicología.

\section{Referencias}

Bateson, M. C. (1979). The epigenesis of conversational interaction: A personal account of research development. En M. Bulowa (Ed.), Before speech. The beginning of interpersonal communication (pp. 63-78). Cambridge: Cambridge University Press.

Beebe, B., Sorter, D., Rustin, J. \& Knoblauch, S. (2003). A comparison of Meltzoff, Trevarthen and Stern. Psychoanalytic Dialogues, 13(6), 777-804.

Blanco, F. (2002). El cultivo de la mente: Un ensayo histórico-crítico sobre la cultura psicológica. Madrid: Antonio Machado.

Castro, J., Pizarroso, N. \& Morgade, M. (2005). La psicologización del ámbito de lo estético entre mediados del siglo XIX y principios del XX. Estudios de Psicología, 26(2), 195-220.

Cross, I. (2003). Música, cultura y evolución. En I. Martinez \& C. Mauleón (Eds.), Actas de la Tercera Reunión Anual 
de la Sociedad Argentina para las Ciencias Cognitivas de la Música. Buenos Aires: SACCoM. CD-ROM.

Deliege, I. \& Sloboda, J. (Eds). (1996). Musical beginnings. Origins and development of musical competence. Oxford: Oxford University Press.

Dissanayake, E. (2000a). Antecedents of the temporal arts in early mother-infant interaction. En N. L. Wallin, B. Merker \& S. Brown (Eds.), The origins of music (pp. 389410). Cambridge, MA: The MIT Press.

Dissanayake, E. (2000b). Art and intimacy. How the arts began. Seattle: University of Washington Press.

Dissanayake, E. (2001). Becoming homo aestheticus: Source of aesthetic imagination in mother-infant interactions. Substance, 94/95, 85-103.

Dissanayake, E. \& Miall, D. (2003). The poetics of babytalk. Human Nature, 14(4), 337-364.

Español, S. (2004). Cómo hacer cosas sin palabras. Gesto y ficción en la infancia temprana. Madrid: Antonio Machado.

Español, S. (2005). Ontogénesis de la experiencia estética. La actitud contemplativa y las artes temporales en la infancia. Estudios de Psicología, 26(2), 139-137.

Español, S. (2006). Las artes del tiempo en psicología. En F. Shifres \& G. Vargas (Eds.), Sonido, imagen y movimiento en la experiencia musical (pp. 9-27). Buenos Aires: SACCoM.

Español, S. (en prensa). Time and movement in symbol formation. En J. Valsiner \& A. Rosa (Eds.), The Cambridge handbook of social-cultural psychology. Cambridge: Cambridge University Press.

Goldman, A. (1993). The psychology of folk psychology. Behavioural and Brain Sciences, 16, 15-28.

Goldman, A. (2000). The mentalizing folk. En D. Sperber (Ed.), Metarrepresentations: An multidisciplinary perspective (pp. 171-196). Oxford: Oxford University Press.

Gómez, J. C. (1996). Second person intentional relations and the evolution of social understanding. Behavioural and Brain Science, 19(1), 129.

Gómez, J. C. (1998). Do concepts of intersubjectivity apply to non-human primates? En S. Brâten (Ed.), Intersubjective, communication and emotion in early ontogeny (pp. 245-259). Cambridge: University Press.

Gómez, J. C. \& Martín-Andrade, B. (2002). Possible precursors of pretend play in non pretend action of captive gorillas. En R. W. Mitchell (Ed.), Pretending and imagination in animals and children (pp. 255-268). Cambridge University Press.

Gomila, A. (2003). La perspectiva de la segunda persona de atribución mental. En A. Duarte \& E. Rabossi (Eds.), Psicología cognitiva y filosofía de la mente (pp. 195-218). Buenos Aires: Alianza.

Gopnik, A. \& Meltzoff, A. N. (1997/1999). Palabras, pensamientos y teorías. Madrid: Visor.

Harris, P. L. (1993). Pretending and planning. En S. BaronCohen, H. Tager-Flusberg \& D. Cohen (Eds.), Understanding other minds: Perspective from autism (pp. 228-246). Oxford: Oxford University Press.

Harris, P. L. (2000/2005). El funcionamiento de la imaginación. Buenos Aires: Fondo de Cultura Económica.

Hobson, R. P. (1993/1995). El autismo y el desarrollo de la mente. Madrid: Alianza.

Imberty, M. (2002). La musica e il bambino. En J. J. Nattiez (Dir.), Enciclopedia della musica (pp. 477-495). Torino: Giulio Einaudi Editore.
Kugiumutzakis, G. (1998). Neonatal imitation in the intersubjective companion space. En S. Brâten (Ed.), Intersubjective, communication and emotion in early ontogeny (pp. 63-88). Cambridge: Cambridge University Press.

Langer. S. (1967). Mind: An essay on human feeling. Vol 1. Baltimore, MD: Johns Hopkins Universities Press.

Lerdahl, F. \& Jackendoff, R. (1983). A generative theory of tonal music. Cambridge, MA: The MIT Press.

Lewkowicz, D. (1992). Infants' response to temporally based intersensory equivalence: The effect of synchronous sounds on visual preferences for moving stimuli. Infant Behavior and Development, 15, 297-324.

Lewkowicz, D. (2000). The development of intersensory temporal perception: An epigenetic systems/limitations view. Psychological Bulletin, 126(2), 281-308.

Lewkowicz, D. (2002). Heterogeneity and heterochrony in the development of intersensory perception. Cognitive Brain Research, 14, 41-63.

Merker, B. (2002, julio). Principles of interactive behavioral timing. En C. Stevens, D. Burham, G. McPherson, E. Schubert \& J. Renwick (Eds.), Proceedings of the 7th International Conference of Music Perception and Cognition (pp. 149-152). Sydney: University of Western Sydney.

Murray, L. \& Trevarthen, C. (1984). Emotional regulation of interactions between two-month-olds and their mothers. En T. Field \& N. Fox (Eds.), Social perception in infants (pp. 177-197). Norwood, NJ: Albex.

Nadel, J. (1996). Early interpersonal timing and the perception of social contingences. Infant Behaviour and Development, 19, 172-202.

Papôusek, M. (1996). Intuitive parenting: A hidden source of musical stimulation in infancy. En I. Deliege \& J. Sloboda (Eds.), Musical beginnings. Origins and development of musical competence (pp. 88-112). Oxford: Oxford University Press.

Rivière, A. (1987/2003). Interacción precoz. Una perspectiva vygotskiana a partir de los esquemas de Piaget. En M. Belinchón, A. Rosa, M. Sotillo \& I. Marichalar (Eds.), Ángel Rivière. Obras escogidas (pp. 109-142). Madrid: Panamericana.

Rivière, A. (1991). Objetos con mente. Madrid: Alianza.

Rivière, A. (1997/2003). Teoría de la mente y metarrrepresentación. En M. Belinchón, A. Rosa, M. Sotillo \& I. Marichalar (Eds.), Ángel Rivière. Obras escogidas (pp. 191-231). Madrid: Panamericana.

Rivière, A. (1999/2003). Desarrollo y educación: El papel de la educación en el diseño del desarrollo humano. En M. Belinchón, A. Rosa, M. Sotillo \& I. Marichalar (Eds.), Ángel Rivière. Obras escogidas (pp. 203-242). Madrid: Panamericana.

Rivière, A. \& Sotillo, M. (1999/2003). Comunicación, suspensión y semiosis humana: Los orígenes de la práctica y de la comprensión interpersonal. En M. Belinchón, A. Rosa, M. Sotillo \& I. Marichalar (Eds.), Ángel Rivière. Obras escogidas (pp. 181-201). Madrid: Panamericana.

Scotto, C. (2002). Interacción y atribución mental: La perspectiva de segunda persona. Análisis Filosófico, 22(2), 135-152.

Shifres, F. \& Español, S. (2004, agosto). Interplay between pretend and music play. Ponencia presentada en $8^{\text {th }}$ International Conference on Music Perception and Cognition, Evanston, Ilinois, EE.UU. 
Sperber, D. (Ed.). (2000). Metarrepresentations: An multidisciplinary perspective. Oxford: Oxford University Press.

Stern, D. (1985/1991). El mundo interpersonal del infante. Una perspectiva desde el psicoanálisis y la psicología evolutiva. Buenos Aires: Paidós.

Stern, D. (2000). Putting time back into our considerations of infant experience: A microdiachronic view. Infant Mental Health Journal, 21(1-2), 21-28.

Thompson, E. (Ed.). (2001). Between ourselves: Second person issues in the study of consciousness. Thorverton, UK: Imprint Academic.

Tooby, J. \& Cosmides, L. (2001). Does beauty build adapted minds? Towards an evolutionary theory of aesthetics, fiction, and arts. Substance, 94/95, 6-27.

Fecha de recepción: Septiembre de 2006.

Fecha de aceptación: Diciembre de 2006.
Treuhb, S. (2000). Human processing predispositions and musical universals. En N. L. Wallin, B. Merker \& S. Brown (Eds.), The origins of music (pp. 427-448). Cambridge, MA: The MIT Press.

Trevarthen, C. (1982). The primary motives for cooperative understanding. En G. Butterworth \& P. Light (Eds.), Social cognition (pp. 77-109). Brighton: Harverster.

Trevarthen, C. (1998). The concept and foundations of infant intersubjectivity. En S. Brâten (Ed.), Intersubjective, communication and emotion in early ontogeny (pp. 1546). Cambridge: Cambridge University Press.

Trevarthen, C. (2000). Musicality and the intrinsic motive pulse: Evidence from human psychobiology and infant communication [Special Issue]. Musica Scientice, 155215.

Wallin, N., Merker, B. \& Brown, S. (Eds.). The origins of music. Cambridge, MA: The MIT Press. 